\title{
Study on the Effect of Mental Health Education on Breastfeeding Behavior in Pregnant and Perinatal Period
}

\author{
Lihui $\mathrm{Si}^{1}$, Shuli Yang, Yan Jia, Wenwen Jian and Qing Yu, \\ Min Wang and Ruixin Lin ${ }^{\text {* }}$ \\ ${ }^{1}$ The Second Hospital of Jilin University, Changchun city Jilin Province, 130012 \\ axddfc@126.com
}

\begin{abstract}
Keywords: Pregnancy and Childbirth, Mental Health Education, Breastfeeding Behavior, Influence Study
\end{abstract}

Abreact. Objective: Carry out maternal health education during pregnancy and childbirth, conduct observation and analysis of maternal breastfeeding behavior. Methods: take 120 pregnant women attending our hospital during the period of February 2017 to January 2018, randomly divide them into control group and observation group. The control group is given only routine nursing intervention while the observation group is given both routine nursing intervention as well as mental health education. After that, we compare the nursing effects including the time gap when breasts start to make milk, postpartum depression and the feeding situation. Compared with the control group, the time gap for breast starting to produce milk is shorter than the control group, and there is a significant difference between the groups $(\mathrm{P}<0.05)$. The percentage of exclusive breastfeeding in the observation group is significantly higher, and the appearance of postpartum depression significantly decreased, the differences between the groups is significant $(\mathrm{P}<0.05)$, and women have postpartum depression are relieved while their mental health education knowledge is strengthened. Conclusion: the implementation of mental health education nursing intervention can help pregnant women control their emotions, enhance their mental capacity, relieve postpartum depression, adapt to this special period and adjust breastfeeding along with higher nursing satisfaction rate and is worthy of clinical significance.

Breastfeeding is the healthiest diet for infants, infants fed by breast milk are proven to have better immunity, intelligence and vitality. It is also believed that breastfeeding can also reduce the mortality rate of infants and young children, especially premature infants, and the health benefits can be extended to adulthood. Breastfeeding is one of the most advocating methods in the World Health Organization and the The United Nations Children's Fund. It is an important process that ensures the healthy growth of babies. Breastfeeding mainly refers to the use of mother's milk to feed their infants ${ }^{[1]}$. Breast milk is considered as a natural food source for infants, breast-feeding is not only important for the infant's late-natal nutrition, growth, and immunity, but it can also prevent maternal postpartum hemorrhage, increase body metabolism, and help reduce complications. Postpartum stage of early lactation and multiple lactation should be considered as as one of the key links in the success of breastfeeding ${ }^{[2-3]}$. However, in recent years, pregnant women are affected by many complex factors such as life, society, psychology, physiology, and spirit. The rate of breast-feeding has shown a significant downward trend, which is not conducive to healthy growth and development of infants. In the clinical department, there are often many mothers who do not produce milk after giving birth, and the newborn needs mother feeding, which is a more worrying issue. Some mothers with a small amount of milk do not cooperate actively or they may also be embarrassed to do so. What's more, it also physically, psychologically affect their life. If it is more serious, it may have a repulsive effect, and it is more likely to trigger a series of negative emotions (depression, etc.). As a result, the curative effect and prognosis will be seriously affected and is not conducive to maternal rehabilitation, causing great psychological burden on the mothers ${ }^{[4-5]}$. Relevant medical workers should pay great attention to this and mental health education plays an important role in maternal prevention and treatment. This article analyzes the effect of mental health education during maternity period on the breast feeding behavior. 


\section{Materials and Methods}

\section{Materials}

From February 2017 to January 2018, 120 women who were admitted to our hospital are selected as study subjects. They are randomly divided into two groups and both control group and observation group has 60 cases. The age of pregnant women in the control group is between 25-40, average age is $(30.3 \pm 4.8)$; the age of pregnant women in the observation group is between $25-41$, average age is (32.5 \pm 4.0$)$. People from both groups are approved by the Hospital Ethics Committee. We then compare general information (gender, age etc.), and the result shows that it does not have statistical significance $(\mathrm{P}>0.05)$ and significant difference but is comparable.

\section{Methods}

The pregnant women in the control group are given the hospital's regular care. The pregnant women in the observation group are given mental health education nursing interventions. The specific measures for mental health education show as follows: Postpartum recovery may lead to pain due to incision, uterine contraction pain, inappropriate maternal breast-feeding, improper breast-feeding, chapped pain, and other physical disorders such as coughing, defecation, getting out of bed, breastfeed depression, poor speech or psychological irritability. To strengthen psychological education, implement targeted maternal comfort, guidance and relaxation methods can help to eliminate maternal tension and fear which is also conducive to maternal and child skin contact (more than half an hour) and early sucking. Caregivers at this time should use scientific theory knowledge and patiently explain the precautions: for example how to avoid pelvic adhesions, proper activities will help the uterus and avoid lochia discharge based on appropriate activities. In addition, we can also take beneficial measures in many ways to reduce the pain, such as postoperative drug analgesia, provide a warm, comfortable and quiet family environment. The room light should be soft so as to transfer maternal pain attention; breast feeding should be adopted while caregivers should help women understand some scientific knowledge as well as operational technologies to cultivate breast feeding habits and assist in neonatal related care; encourage maternal postpartum breastfeeding to recognise and encourage positive behaviors of mothers, strengthen their self-confidence, increase pain threshold, and try to reduce or avoid the pain caused by a series of psychological discomfort. Maternal postpartum women need to recover from the discomfort during gestational and cesarean section and undergo a period of dependency, dependence-independence and independence ${ }^{[8]}$. The experience of childbirth delivery includes high enthusiasm, hope, happiness, satisfaction, happiness, optimism, depression and anxiety. Women giving birth will feel empty and being a mother also bring about a series of responsibilities. At this point, caregivers should take the initiative to contact the mothers along with full enthusiasm and demonstrate this in language as well as behavior so as to create mutual trust. Moreover, family members should be encouraged to care about the mother and provide good condition to take care of both the mother and baby to avoid adverse mental stimulation. To create a safe, warm, harmonious and comfortable family environment for mothers, help them adapt to being a mother as well as enhance maternal and child emotions are also of prominent significance ${ }^{[9]}$.

\section{Evaluation indicators}

The two groups are evaluated according to the Hamilton Depression Rating Scale (HAMD), which is a criterion for assessing the efficacy of depression; the time when breast start to produce milk as well as the breastfeeding condition should also be recorded.

\section{Statistical processing}

For the two groups treated based on different means, we use SPSS 14.0 statistical software to put the data and carry out statistical analysis as well as comparison. The measurement data is expressed as mean \pm standard deviation $(x \pm s)$, the $t$ is used for test; count data is expressed as percentage $(\%)$. If $\mathrm{P}<0.05$, it shows that there is a significant difference and is worthy of statistical significance. 


\section{Results}

The time gap when breast starts to produce milk

According to the results in Table 1, the results of the observation group and the control group are compared. The pregnant women in the observation group have a shorter gap for breasts to produce milk and there is a significant difference $\mathrm{t}(\mathrm{P}<0.05)$. See Table 1 for details.

Table 1 The time gap when breast starts to produce milk

\begin{tabular}{cccccc}
\hline Group & Examples & $1 \mathrm{~h}(\mathrm{n})$ & $1-6 \mathrm{~h}(\mathrm{n})$ & $6-24 \mathrm{~h}(\mathrm{n})$ & $>24 \mathrm{~h}$ \\
\hline $\begin{array}{c}\text { Control group } \\
\begin{array}{c}\text { Observation } \\
\text { group }\end{array}\end{array}$ & 60 & $8(13.3 \%)$ & $17(28.3 \%)$ & $23(38.3 \%)$ & $12(20 \%)$ \\
\hline
\end{tabular}

\section{Breast feeding percentage of two groups}

Pure breast feeding percentage in observation group after intervention is much higher than the one in control group and the significant difference is statistically meaningful $(\mathrm{P}<0.05)$, see table 2.

Table 2. Breast feeding percentage of two groups $(\%)$

\begin{tabular}{ccccc}
\hline Group & $\mathrm{n}$ & $\begin{array}{c}\text { Pure breast feeding } \\
(\mathrm{n})\end{array}$ & $\begin{array}{c}\text { Artificial feeding } \\
(\mathrm{n})\end{array}$ & $\begin{array}{c}\text { Breast feeding and } \\
\text { diary products }(\mathrm{n})\end{array}$ \\
\hline $\begin{array}{c}\text { Observation } \\
\text { group }\end{array}$ & 60 & $44(73.3 \%)$ & $7(11.7 \%)$ & $9(15 \%)$ \\
Control group & 60 & $24(40.0 \%)$ & $16(26.7 \%)$ & $20(33.3 \%)$ \\
\hline
\end{tabular}

\section{Satisfaction degree}

The satisfaction of the two groups of patients after the health instruction intervention is evaluated and the results are shown in Table 3. The satisfaction of the patients in the observation group is 93.3\%; the satisfaction in the control group is only $61.7 \%$, and the satisfaction of the observation group improved by $31.6 \%$, there is a significant difference in satisfaction between groups $(\mathrm{P}<0.05)$.

Table 3 Satisfaction survey results (n)

\begin{tabular}{cccccc}
\hline Group & $\mathrm{n}$ & $\begin{array}{c}\text { Satisfactio } \\
\mathrm{n}\end{array}$ & $\begin{array}{c}\text { Partly } \\
\text { satisfaction }\end{array}$ & $\begin{array}{c}\text { Dissatisfactio } \\
\mathrm{n}\end{array}$ & $\begin{array}{c}\text { Satisfaction } \\
\text { percentage }(\%)\end{array}$ \\
\hline $\begin{array}{c}\text { Observation } \\
\text { group }\end{array}$ & 60 & 33 & 23 & 4 & 93.3 \\
Control group & 60 & 21 & 16 & 23 & 61.7 \\
\hline
\end{tabular}

\section{HAMD evaluation result}

From the results, we can see that the scores of the HAMD scores before and after the intervention are compared between the two groups. After the intervention, the HAMD scores of the observation group show a significant decrease compared with those before the intervention. Compared with the control group, the difference between the groups is significant $(\mathrm{P}<0.05)$, as shown in Table 4.

Table 4. HAMD evaluation results of two groups

\begin{tabular}{cccccc}
\hline Group & $\mathrm{n}$ & $\begin{array}{c}\text { Before treatment } \\
\text { evaluation }\end{array}$ & After 1 month & $\begin{array}{c}\text { After 2 } \\
\text { months }\end{array}$ & $\begin{array}{c}\text { After 3 } \\
\text { months }\end{array}$ \\
\hline $\begin{array}{c}\text { Observation } \\
\text { group }\end{array}$ & 60 & $35.5 \pm 2.9$ & $24.1 \pm 3.1$ & $19.7 \pm 2.9$ & $18.1 \pm 1.6$ \\
Control group & 60 & $34.4 \pm 2.5$ & $31.6 \pm 2.9$ & $27.3 \pm 2.7$ & $25.0 \pm 1.0$ \\
\hline
\end{tabular}

\section{Conclusion}

In clinical practice, obstetrical psychological health education is considered as a professional and highly theoretical preventive measure, which has a direct relationship with the recovery of pregnant women, and is conducive to improving the satisfaction of pregnant women to hospitals. Obstetric pregnant women, as a special group of society nowadays are experiencing more and more pressure 
and diseases with the social development. Mental health education based on the actual situation of patients along with targeted psychological guidance will help to improve compliance. Helping pregnant women to recover and give good breast feeding we should improve their life, increase their satisfaction and decrease side effects, reduce unhappiness all play a positive role in improving breastfeeding. Therefore, the adoption of appropriate mental health education intervention will contribute better results.

From the results of the study, it can be seen that in the breastfeeding behavior, 55 women start to produce milk in 24 hours, reaching to $91.7 \%$ while there are only 40 people start to produce milk in 24 hours in the control group, and the percentage is only $80 \%$. Therefore, we can see that the time gap to produce breast milk in the control group is obviously lower than the observation group, indicating that mental health education has a significant effect on maternal breastfeeding behavior. After the intervention of mental health education, the exclusive breastfeeding rate in the observation group is significantly higher than that in the control group. The difference between the two groups is statistically significant $(\mathrm{P}<0.05)$. The satisfaction rate of the observation group is $93.3 \%$; the control group's satisfaction is only $61.7 \%$, satisfaction in the observation group increases by $31.6 \%$, and there is a significant difference in satisfaction between the groups $(\mathrm{P}<0.05)$. After the intervention, the HAMD scores in the observation group show a significant decrease compared with that before the intervention. Compared with the control group, the decrease is significant and the difference between groups is significant $(\mathrm{P}<0.05)$. It shows that active and effective mental health education, such as knowledge education as well as psychological counseling, is provided for pregnant women to increase awareness of the importance of breastfeeding and to improve maternal breastfeeding behavior.

In summary, this study does not only select appropriate prevention measures in the maternal and childbirth period interventions, but also take related mental health education activities in the treatment. After the implementation, the negative emotion of pregnant women is effectively alleviated and they are no longer in an extreme depressed and stressful mental state. As a result, the breastfeeding behavior is improved, which can contribute to the recovery of the mother and the growth of the newborn. Therefore, it has a positive guiding significance in clinical practice and is worthy of further reference, which can accumulate more experience for clinical work in the future and better serve patients.

\section{References}

[1] Li Chaohong. Practice and discussion on health education model of hospital in pregnant and perinatal period Chinese Journal of Health Education[J]Chinese Journal of Health Education, 2016, 32(11):1052-1055.

[2] Zhang Jinjia, Liu Xiaohong. Effects of maternal factors on the development of infants' behavior[J].Chinese Journal for Clinicians, 2017, 45(1): 26-28.

[3] Zhang Xuan. Evaluation and analysis of health education effect in pregnant and perinatal period in Sichuan Province[J]. Maternal and Child Health Care of China. 2015, 30(26): 4435-4436.

[4] Mei Ru, Wang Yajun. Analysis of pregnant women's psychological depression anxiety and related influencing factors[J].Chinese Journal of Clinical Research, 2015, 28(6): 752-754.

[5] Han Mingfang, Wang Liping, Wang Xiaoyun etc. Effect of situational health education on the formation of maternal breast feeding ability[J]. Guangdong Medical Journal, 2013, 34(21): 3367-3368.

[6] Luo Juan, Zheng Jiayong. Impact of health education on maternal health knowledge, behavior and skills[J]. Maternal and Child Health Care of China, 2016, 31(15): 3137-3140.

[7] Tang Jinamei, Yan Liying. The influence of loving education of relatives and accompanying volunteers on Parturient's delivery mode and puerperal behavior[J]. Medical Journal of West China, 2015, 27(9): 1406-1408.

[8] Chen Huali, Xiang Longquan, Chen Juanli etc. Effects of integrated management of pregnancy and childbirth on maternal outcomes and breastfeeding [J]. Journal of Nursing Administration, 2014, 14(7): 502-503 
[9] Wang Bo, Tai Fadao. Effects of early parental absence on offspring behavior and neuroendocrine in mammals[J]. Journal of Mammals, 2013, 33(4): 367-376.

[10] Li Chunping. Effect of clinical pathway health education on compliance of breastfeeding[J].Chinese Journal of Health Education, 2016, 32(7): 655-657.

[11] Mao Hongni, Zhang Xiaoqian, Meng Qingzhong. The effect of mental health education on breastfeeding behavior in pregnant and perinatal period $[\mathrm{J}]$. China Journal of Health Psychology, 2018, 26(1):58-62. 